\title{
CRIANÇAS COM DISTÚRBIOS DO SONO APRESENTAM ALTERAÇÃO NO POSICIONAMENTO DA CABEÇA E DO OMBRO?
}

\author{
DO CHILDREN WITH SLEEP DISORDERS PRESENT CHANGE IN \\ HEAD AND SHOULDER POSITIONING?
}

Maria Eduarda Radigonda Paranzini (ORCID: 0000-0001-9260-7392)

Paola Janeiro Valenciano (ORCID: 0000-0002-8363-0475) ${ }^{2}$

Celita Salmaso Trelha (ORCID: 0000-0001-5643-9002) ${ }^{3}$

Dirce Shizuko Fujisawa (ORCID: 0000-0001-8427-2860) ${ }^{3}$

Autor Correspondente

Maria Eduarda Radigonda Paranzini: E-mail: duda.radigonda@gmail.com

\footnotetext{
${ }^{1}$ Curso de Graduação em Fisioterapia. Universidade Estadual de Londrina

2 Programa de Pós-Graduação em Ciências da Reabilitação. Universidade Estadual de Londrina

${ }^{3}$ Departamento de Fisioterapia. Universidade Estadual de Londrina
}

a) Este trabalho é resultante do Trabalho de Conclusão de Curso de Maria Eduarda Radigonda Paranzini, intitulado "Crianças com distúrbios do sono apresentam alteração no posicionamento da cabeça e do ombro?", apresentado ao Curso de Graduação em Fisioterapia da Universidade Estadual de Londrina em 2018.

\begin{abstract}
RESUMO
Objetivo: verificar a correlação entre distúrbios de sono e alteração do posicionamento da cabeça e do ombro em crianças escolares. Metodologia: trata-se de estudo transversal, com amostra de conveniência de 40 crianças entre 8 e 12 anos. Foram coletados dados pessoais e antropométricos. A avaliação postural foi por meio da Fotogrametria, com variáveis relacionadas com o posicionamento da cabeça e do ombro nas vistas frontal e sagital. A qualidade do sono foi avaliada pelo Questionário de Hábitos de Sono das Crianças (CSHQ-PT). O teste Shapiro-Wilk foi utilizado para verificar a normalidade dos dados. Para análise de correlação, foi utilizado o Coeficiente de Correlação de Spearman. A significância foi estabelecida em 5\%. Resultados: as alterações encontradas foram referentes ao alinhamento horizontal dos acrômios, indicando inclinação à esquerda no plano frontal. Na vista sagital, os resultados evidenciaram a protusão dos ombros bilateralmente. A correlação ocorreu entre a ansiedade associada ao sono e alinhamento horizontal da cabeça (vista lateral direita), e entre a resistência em ir para a cama e o ângulo dos ombros. Quanto maior a pontuação da ansiedade, menor o grau de alinhamento horizontal da cabeça (anteriorização); e quanto maior a resistência de ir para a cama, maior o ângulo dos ombros (protusão). Conclusão: Na prática clínica, deve-se ter atenção especial à qualidade do sono e à evolução postural na infância.
\end{abstract}

Palavras-chave: Criança; Distúrbios do sono e da vigília; Postura.

\begin{abstract}
Objective: the objective of the study was to verify the correlation between sleep disorders and the alteration of head and shoulder positioning in school children. Methodology: this is a cross-sectional study with a convenience sample of 40 children between 8 and 12 years old. Anthropometric and personal data were collected. The postural evaluation was by means of photogrammetry, with variables related to head and shoulder positioning in frontal and sagittal view. The quality of sleep was evaluated by "Children's Sleep Habits Questionnaire" (CSHQ-PT). Shapiro-Wilk test was used to verify the normality of the data. Spearman Correlation Coefficient was used to study the correlation analysis. A statistical significance of 5\% was adopted. Results: the alteration found were referred to the acromion's horizontal alignment, indicating inclination to the left in the frontal plan. In the sagittal view, the results have evidenced shoulder protrusion bilaterally. The results that obtained correlation were between anxiety associated with sleep and the right horizontal head alignment. It was also found correlation between resistance in going to bed and shoulder angle. The higher the anxiety score, the lower the degree of horizontal alignment of the head (anteriorization) and the higher the resistance to go to bed, the greater the angle of the shoulders (protrusion). Conclusion: in clinical practice, special attention should be paid to sleep quality and postural evolution in childhood.
\end{abstract}

Keywords: Child; Sleep wake disorders; Posture. 


\section{INTRODUÇÃO}

O sono, que consiste em uma necessidade biológica e vital, é um fator importante na saúde da criança ${ }^{1}$. Os hábitos do sono, que envolvem o padrão do ciclo sono/vigília e a quantidade diária necessária, são modificados conforme a idade, havendo grande diferença entre o sono de adultos e de crianças ${ }^{1,2 .}$ Existem variações em relação à necessidade do sono no período pré-escolar: as crianças tendem a dormir, em média, de 12 a 13 horas por dia; e as escolares, dormem de 10 a 12 horas por dia ${ }^{1}$.

Crianças em diferentes faixas etárias podem manifestar distúrbios do sono; cerca de $30 \%$, em algum momento do desenvolvimento, irão apresentar algum sintoma ${ }^{2}$. Tais distúrbios influenciam negativamente a qualidade do sono da criança $\mathrm{e}$, consequentemente, interferem no crescimento e desenvolvimento ${ }^{1}$. Os distúrbios do sono se diferenciam conforme a idade, e as queixas podem ser diferenciadas em insônia, que é a dificuldade para iniciar ou manter o sono, parassonias, que representam as dificuldades respiratórias que ocorrem durante o sono e, também, sonolência diurna excessiva ${ }^{1,2}$. Os distúrbios de sono mais frequentes na pré-escola são o despertar e o terror noturno; e na adolescência, a insônia e o sonambulismo ${ }^{1}$.

Os desvios posturais são problemas importantes na população infantil e adolescência, já que estão em desenvolvimento corporal, geralmente, decorrentes da má postura ${ }^{3}$. Entre 7 e 12 anos de idade, surgem adaptações funcionais resultantes dos desvios da coluna vertebral, pois há intensa mobilidade e a postura se adapta às atividades. Durante o início da vida escolar, o comportamento postural da criança é tido como responsável pelos vícios posturais adquiridos, considerando a evolução da postura ereta, as condições anatômicas, a coluna vertebral e as relações da criança com o meio em que vive 4 .
A posição frequentemente adotada para repouso, em que há mínima influência da gravidade, e que coordena a musculatura flexora e extensora em alongamento é a postura semifletida de todos os segmentos no decúbito lateral. Tal posição contribui para maior sensação de relaxamento do indivíduo ${ }^{5}$. Além da colaboração da postura para a qualidade de relaxamento dos segmentos corporais, sabe-se que o tempo insuficiente de sono favorece a prevalência de algias. Os adultos que dormem menos de oito horas por dia apresentam maior prevalência de dor cervical ${ }^{6}$.

Ao nosso conhecimento, os estudos que relacionam a qualidade do sono com a postura da cabeça e do ombro em crianças são escassos. Assim, este estudo teve como objetivo verificar a correlação entre distúrbios do sono e alteração no posicionamento da cabeça e do ombro em crianças escolares. A hipótese é de que haja correlação positiva entre distúrbios do sono e alterações no posicionamento da cabeça e dos ombros.

\section{METODOLOGIA}

Trata-se de estudo transversal, com amostra de conveniência, participantes entre 8 e 12 anos de idade, de ambos os sexos, inscritos voluntariamente no projeto de pesquisa "Exercícios do método Pilates em solo: efeitos no alinhamento, controle postural, sistema respiratório e na qualidade do sono em crianças saudáveis".

Os critérios de inclusão foram crianças que não realizassem programa de exercícios físicos nos últimos seis meses, além da educação física, e com desenvolvimento típico. Foram excluídas crianças com doenças crônicas, comprometimentos 
musculoesqueléticos, cirurgias prévias recentes, incapacidade de manter a posição ortostática, déficit físico e/ou sensorial, queixa de tontura ou vertigem, déficit de atenção ou de compreensão e uso medicamento contínuo.

\section{Coleta de dados}

$\mathrm{Na}$ avaliação dos dados antropométricos, foi utilizada fita métrica $(150 \mathrm{~cm})$ para mensuração da estatura com as crianças eretas, joelhos em extensão, pés juntos, braços soltos ao longo do corpo e tornozelos, região glútea e ombros em contato com a parede. A massa corporal foi obtida na balança digital (Omron, HN-289), capacidade máxima de $150 \mathrm{~kg}$ e mínima de 5 $\mathrm{kg}$; as crianças estavam sem sapatos ou meias, posição ortostática com os membros ao longo do corpo, posicionadas no centro da balança e olhando à frente.

\section{Questionário de Hábitos de Sono das Crianças (CSHQ-PT)}

O Questionário de Hábitos de Sono das Crianças (CSHQ-PT), validado no Brasil, em português, por Silva et al. ${ }^{7}$, foi respondido pelos pais, com o intuito de avaliar o comportamento do sono durante a semana anterior ou uma semana típica mais recente. A frequência do comportamento do sono é classificada em uma escala de três pontos, como "habitualmente" (cinco a sete vezes por semana, totalizando três pontos), “às vezes” (duas a quatro vezes por semana, totalizando dois pontos) ou "raramente" (zero a uma vez por semana, totalizando um ponto).

\section{Fotogrametria}

A colocação de marcadores nos pontos anatômicos foi realizada por avaliador único e treinado, conforme tutorial de Ferreira8. A análise das imagens também foi realizada por avaliador único e treinado para o uso do programa SAPo (versão 0,68$)^{8}$. A postura foi registrada com câmera digital da marca Sony ${ }^{\circledR}$ de 14,1 Megapixels (Cyber-shot DSC-W320), tripé de alumínio da marca WF® (wt-3510A). A câmera digital ficou posicionada sobre o tripé, altura de $90 \mathrm{~cm}$ do solo, distância de $300 \mathrm{~cm}$ da criança e $350 \mathrm{~cm}$ da parede. Para coleta das imagens, os participantes foram instruídos a permanecer na posição ortostática sobre o tapete, em sua postura usual e confortável, com os braços ao longo do corpo e os pés levemente afastados, com o peso distribuído igualmente, com olhar à frente 9 . As seguintes medidas foram utilizadas na vista anterior:

- Alinhamento horizontal da cabeça: formada pelo ângulo entre os marcadores no tragus à direita e esquerda e a horizontal, valores positivos e negativos indicam inclinação à direita e à esquerda respectivamente ${ }^{10}$.

- Alinhamento horizontal dos acrômios: obtido pelo ângulo entre os marcadores nos acrômios à direita e à esquerda e a horizontal, valores positivos e negativos indicam inclinação à direita $\mathrm{e}$ à esquerda respectivamente ${ }^{10}$.

$\mathrm{Na}$ vista lateral direita e esquerda:

- Alinhamento horizontal da cabeça: formado pelo ângulo entre os marcadores no processo espinhoso de C7, tragus e a horizontal, valores menores que $50^{\circ}$ indicam a anteriorização da cabeça $a^{11,12}$.

A análise por medidas livres foi realizada na vista lateral para o ângulo dos ombros, formado pelo ângulo entre processo espinhoso de C7, acrômio e a horizontal, para identificar a protração (ângulos menores que $52^{\circ}$ ) e retração do ombro ${ }^{12}$.

\section{Análise dos dados}

Os dados foram analisados no programa GraphPadPrism ${ }^{\circledR} 6$. A distribuição da normalidade foi avaliada pelo teste Shapiro-Wilk. Os dados estão apresentados em média e desvio-padrão ou mediana e 
intervalos interquartílicos [25-75]. A significância foi estabelecida em $5 \%$.

A correlação avaliada pelo Coeficiente de Correlação de Spearman, classificados conforme os seguintes parâmetros: coeficientes menores de 0,30 - fraca magnitude; entre 0,30 e 0,50 - moderada magnitude; e maiores que 0,50 - forte magnitude. As correlações consideradas foram as que obtiveram significância estatística $(p \leq 0,05)$ e de moderada a forte magnitude ${ }^{13}$.

O estudo foi aprovado pelo Comitê de Ética em Pesquisa da Universidade Estadual de Londrina (Parecer $\mathrm{n}^{\circ}$ 2.683.370). Os pais ou responsáveis das crianças foram esclarecidos quanto ao estudo, autorizando a participação do menor por meio do Termo de Consentimento Livre e Esclarecido.

\section{RESULTADOS}

Participaram 40 crianças, com maior proporção do sexo feminino $(85 \%)$. A caracterização da amostra está descrita na tabela 1. A média do IMC foi de $19 \mathrm{~kg} /$ $\mathrm{m}^{2}$, que indica sobrepeso para a idade, segundo a Organização Mundial da saúde (OMS) (eutrofia valores entre 13,1-18,3 para meninas e 13,6-18,2 para meninos) $)^{14}$.

Tabela 1. Caracterização da amostra. Londrina, 2018

\begin{tabular}{lc}
\hline \multicolumn{1}{c}{ Variáveis } & $\begin{array}{c}\text { Crianças } \\
(\mathbf{n = 4 0 )}\end{array}$ \\
\hline Idade & $9[8,2-11,0]$ \\
Sexo masculino $(\%)$ & $6(15)$ \\
Sexo feminino $(\%)$ & $34(85)$ \\
Massa corporal $(\mathrm{kg})$ & $41,5 \pm 11,5$ \\
Estatura $(\mathrm{cm})$ & $143,8 \pm 10,3$ \\
IMC $\left(\mathrm{kg} / \mathrm{m}^{2}\right)$ & $19[16,5-22,6]$ \\
\hline
\end{tabular}

IMC: Índice de Massa Corporal; Valores apresentados em média e desvio-padrão ( \pm ) ou mediana e intervalos interquartílicos [25-75]. 
O CSHQ-PT, aplicado para verificar a qualidade do sono das crianças, apresentava oito diferentes escalas, que foram analisados separadamente (tabela 2).

Tabela 2. Resultados do Questionário de Hábitos de Sono das Crianças (CSHQ-PT). Londrina, 2018

\begin{tabular}{lc}
\hline \multicolumn{1}{c}{ Variáveis } & $\begin{array}{c}\text { Crianças } \\
(\mathbf{n = 4 0 )}\end{array}$ \\
\hline Tempo total de sono diário (minutos) & $540[502,5-600,0]$ \\
Resistência em ir para cama (subescala 1) & $10[9,0-11,0]$ \\
Início do sono (subescala 2) & $2[1,0-2,0]$ \\
Duração do sono (subescala 3) & $7[6,0-7,0]$ \\
Ansiedade associada ao sono (subescala 4) & $5[4,0-7,0]$ \\
Despertares noturnos (subescala 5) & $3[3,0-5,0]$ \\
Parassonias (subescala 6) & $8,5[8,0-10,0]$ \\
Perturbações Respiratórias do Sono (subescala 7) & $3[3,0-4,0]$ \\
Sonolência diurna (subescala 8) & $13,7 \pm 2,5$ \\
\hline
\end{tabular}

A análise postural foi realizada nas vistas frontal e sagital; e os valores, apresentados em graus (tabela 3). Na vista frontal, a mediana referente ao alinhamento horizontal da cabeça foi de $0^{\circ}$, do alinhamento horizontal dos acrômios foi de $-0,8$, sendo que valor negativo indica inclinação à esquerda no plano frontal; e positivo, inclinação à direita. Já na vista sagital, o alinhamento horizontal da cabeça à direita e à esquerda foi de $47,9^{\circ}$ e $48,5^{\circ}$ respectivamente.

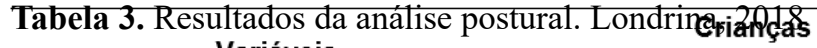
Variáveis

$(n=40)$

\begin{tabular}{lc}
\hline Vista Frontal & \\
AHC (graus) & $0[-1,4-2,2]$ \\
AHA (graus) & $-0,8 \pm 2,4$ \\
& \\
Vista Sagital & $47,9 \pm 5,4$ \\
AHC à direita (graus) & $48,5 \pm 4,7$ \\
AHC à esquerda (graus) & $17,9 \pm 9,1$ \\
Ângulo do ombro direito (graus) & $17,3 \pm 9,3$
\end{tabular}

AHC: Alinhamento horizontal da cabeça; AHA: Alinhamento horizontal dos acrômios; Valores apresentados em média e desvio-padrão $( \pm)$ ou mediana e intervalos interquartílicos [25-75]. 
Todas as variáveis do CSHQ-PT foram utilizadas na análise de correlação com os dados obtidos na avaliação postural. Verificou-se correlação entre a ansiedade associada ao sono e alinhamento horizontal da cabeça à direita, que são inversamente proporcionais (figura 1), também entre a resistência em ir para a cama e o ângulo dos ombros, que são diretamente proporcionais (figura 2).

Figura 1. Correlação entre ansiedade associada ao sono e alinhamento horizontal da cabeça. Londrina, 2018

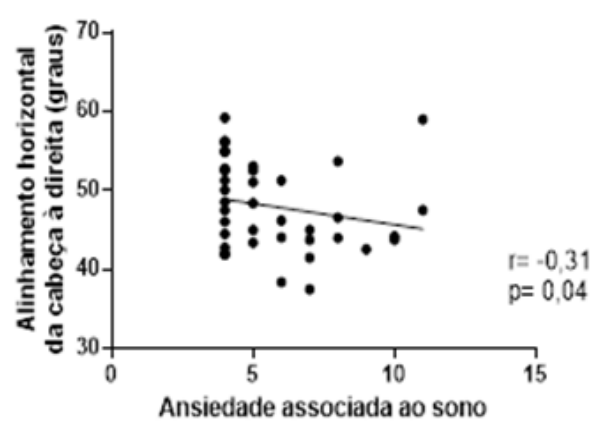

Figura 2. Correlação entre a resistência em ir para cama e ângulos do ombro. Londrina, 2018
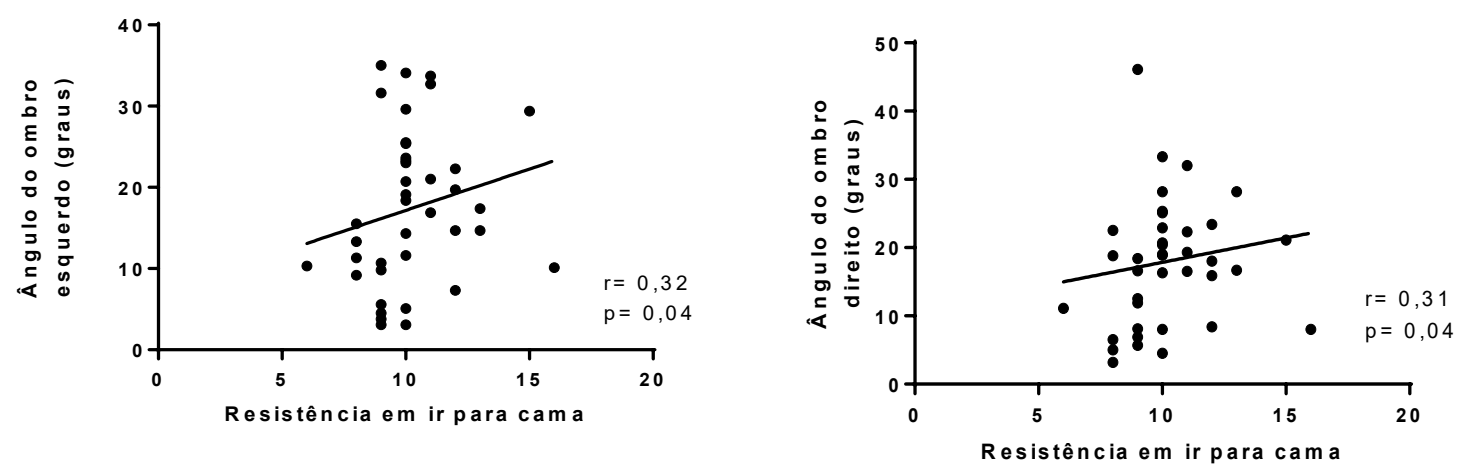
DISCUSSÃO

A média do tempo total de sono diário foi de 540 minutos, a National Sleep Foundation 15 recomenda que crianças em idade escolar devem dormir de 540 a 660 minutos ( 9 a 11 horas). Portanto, a média total de sono diário está dentro do limite inferior de normalidade para a faixa etária. Entre as variáveis do questionário, a maior pontuação foi relacionada com a sonolência diurna. Segundo Felden e colaboradores $16,20 \%$ a $30 \%$ de crianças e adolescentes apresentam distúrbios de sono, em que também a duração inadequada do sono diário e a sonolência diurna podem resultar em maior incidência de hábitos sedentários nessa população. Em relação ao tempo de sono diário, Sedrez et al. ${ }^{17}$ destacaram que o tempo de sono inferior a sete horas pode acarretar alterações posturais, haja vista ser medida de proteção contra os hábitos posturais inadequados. Não há valores de corte específico para a interpretação de cada item da subescala que permita identificar qual a pontuação limite para considerar a qualidade do sono boa ou ruim. Entretanto, ao somar todas as pontuações das subescalas do CSHQ-PT (subescala 1 a 8), há o chamado Índice de Perturbação do Sono, que Silva et al. ${ }^{18}$ sugeriram que, para crianças portuguesas, fosse considerado o ponto de corte de valor $>48$. No presente estudo, a pontuação foi de 52,2 , ou seja, as crianças apresentaram problemas relacionados com o sono ${ }^{18}$.

O período da infância e adolescência é caracterizado por atividades que os mantêm por longo período na postura sentada; tal situação, associada ao sedentarismo, colabora para o desenvolvimento de alterações posturais ${ }^{17}$. Esses fatores podem justificar as alterações encontradas nos resultados da análise postural, visto que, segundo as referências do Protocolo SAPo, o parâmetro normal para alinhamento horizontal de cabeça e alinhamento horizontal dos acrômios é igual a $0^{\circ}$, sendo observada alteração no resultado do alinhamento horizontal dos acrômios $(-0,8 \pm 2,4) 8$. Santos et al. ${ }^{19}$ analisaram a ocorrência de desvios posturais em escolares, e identificaram que a assimetria entre os ombros é consequente ao uso de mochilas escolares de maneira inadequada. A mochila carregada pelos estudantes, em sua maioria, está acima do peso recomendado, o que acarreta compensação postural ${ }^{20}$.

$\mathrm{Na}$ vista lateral, valores menores que $50^{\circ}$ indicam a anteriorização da cabeça, e valores inferiores a $52^{\circ}$ indicam a protração dos ombros ${ }^{12}$. Os resultados indicam a presença de alteração no ângulo dos ombros, considerando-se a discrepância entre os valores encontrados e o valor padrão, evidenciando protração bilateralmente. Santos et al. ${ }^{19}$ também evidenciaram que essa alteração pode ser considerada fisiológica no desenvolvimento infantil, visto que a protração se associa à abdução escapular por meio da ação de grupos musculares que promovem a rotação medial do úmero e tração anterior do ombro, porém essa alteração pode diminuir após os 10 anos de idade.

O estudo de Detsch et al. ${ }^{21}$ mostram prevalência das alterações posturais em escolares na vista lateral e anteroposterior, que corroboram os achados do presente estudo, que podem estar associadas aos hábitos posturais inadequados. 
Também houve maior prevalência de alterações posturais em escolares que assistiam à TV por mais de dez horas semanais, quando comparada às que não assistiam ou que assistiam por tempo menor ${ }^{21}$. Sedrez et al. ${ }^{17}$ encontraram que as alterações posturais estavam associadas à postura inadequada ao sentar e à permanência do uso do computador por quatro horas ou mais. Além disso, a postura adotada pelas crianças ao sentar acompanhava o padrão flexor de tronco, favorecendo os desvios posturais. Noll et al. ${ }^{22}$ identificaram alta prevalência de posturas inadequadas nas atividades diárias de sentar para escrever, sentar em banco, sentar ao utilizar o computador e pegar objeto no chão. Muitas crianças permaneciam tempo maior que quatro horas diárias sentadas de maneira inadequada, utilizando o computador ou assistindo à TV, o que, somado com a média de horas diárias que o escolar permanecia sentado durante os períodos de ensino, resulta em atividade sedentária, prejudicial ao padrão postural $^{22}$.

Os resultados apontaram correlações entre a resistência em ir para a cama e o ângulo dos ombros, que pode estar relacionado com os hábitos e rotinas para adormecer. Rangel et al. ${ }^{23}$ encontraram associação com algumas variáveis dos hábitos e rotinas para adormecer, como a necessidade da presença de um familiar no quarto ou na cama, a visualização de televisão e o uso de videojogos, que interferem na qualidade do sono. A resistência em ir para a cama pode estar relacionada com as atividades atrativas, como as com jogos eletrônicos ou assistir à televisão, que podem gerar esse posicionamento de ombros em protusão.

Também houve associação entre a ansiedade associada ao sono com o alinhamento horizontal da cabeça à direita. A hipótese de a mesma associação com o alinhamento à esquerda não ter sido identificada é que as crianças tenham modificado a postura no momento da avaliação, porém, nas duas vistas, havia anteriorização da cabeça, o que reforça a presença e a preocupação com essa alteração. Ainda, quanto maior a pontuação da ansiedade associada ao sono, menor o grau de alinhamento horizontal da cabeça, ou seja, maior a anteriorização da cabeça.

$\frac{8}{\text { Paranzini et al }}$

A limitação do estudo foi a amostra de conveniência, no entanto, foi possível identificar a correlação entre os distúrbios do sono e o posicionamento da cabeça e do ombro. Sugere-se que outros estudos sejam realizados com tamanho amostral maior e que avaliem os hábitos posturais e de vida com relação à qualidade do sono.

\section{CONCLUSÃO}

A correlação entre os distúrbios do sono e o posicionamento da cabeça e dos ombros foi identificada: quanto maior a pontuação da ansiedade associada ao sono, maior a anteriorização da cabeça; e quanto maior a resistência de ir para a cama, maior a protusão dos ombros. As correlações entre a qualidade do sono e a postura evidenciam a importância na prática clínica de considerar ambos os aspectos em relação à promoção de saúde dos escolares.

\section{REFERÊNCIAS}

1. Maia I, Pinto F. Hábitos de sono. Rev Hosp Crianç Maria Pia. 2008;17(1):9-11.

2. Pessoa JHL. Distúrbios do sono da criança: abordagem pediátrica. Ped Mod. 2003;49(2):73-79.

3. Xavier CA, Bianchi DM, Lima AP, Silva IL, Cardoso F, Beresford H. Uma Avaliação Acerca da Incidência de Desvios Posturais em Escolares. Rev Meta: Avaliação. 2011;3(7):81-94.

4. Oshiro VA, Ferreira PG, Da Costa RF. Alterações posturais em escolares: uma revisão da literatura. Rev Bras Ciênc Saúde. 2007;3(13):15-22.

5. Santos A. A biomecânica da coordenação motora. São Paulo: Summus; 2002. 
6. Fernández-De-Las-Peñas $\mathrm{C}$, Hernández-Barrera V, Alonso-Blanco C, Palacios-Ceña D, Carrasco-Garrido P, Jiménez-Sánchez S, et al. Prevalence of neck and low back pain in community-dwelling adults in Spain. Spine. 2011;36(3):213-219.

7. Silva FG, Silva CR, Braga LB, Serrao Neto A. Portuguese Children's Sleep Habits Questionnaire- validation and cross-cultural comparison. J Pediatr (Rio J). 2014;90(1):78-84.

8. Ferreira EAG. Postura e controle postural: desenvolvimento e aplicação de método quantitativo de avaliação postural [tese]. São Paulo: Faculdade de Medicina, Universidade de São Paulo; 2005.

9. Dolphens M, Cagnie B, Coorevits P, Vleeming A, Danneels L. Classification System of the Normal Variation in Sagittal Standing Plane Alignment. Spine. 2013;38(16):1003-1012.

10. Ribeiro AFM, Bergmann A, Lemos T, Pacheco AG, Russo MM, Oliveira LAS, et al. Reference Values for Human Posture Measurements Based on Computerized Photogrammetry: A Systematic Review. J Manipulative Physiol Ther. 2017;40(3)156-168.

11. Ruivo RM, Pezarat-Correia P, Carita AI. Cervical and shoulder postural assessment of adolescents between 15 and 17 years old and association with upper quadrant pain. Braz J Phys Ther. 2014;18(4):364-371.

12. Ruivo RM, Pezarat-Correia P, Carita AI. Intrarater and interrater reliability of photographic measurement of upper-body standing posture of adolescents. J Manipulative Physiol Ther. 2015;38(1):74-80.

13. Maciel APF, Pimenta HB, Caldeira AP. Qualidade de vida e adesão medicamentosa para pessoas hipertensas. Acta Paul Enferm. 2016;29(5):542-548.

14. Food and Nutrition Technical Assistance. Tabelas de IMC para crianças e adolescentes 5-18 anos de idade, tabelas de IMC-para idade, e tabelas de IMC para adultos não grávidas, não lactantes $\geq 19$ anos de idade [Internet]. Washington, DC: FANTA; jan 2013 [acessado 2018 nov 3]. Disponível em: https://www.fantaproject. org/sites/default/files/resources/FANTA-BMI-charts-Janeiro\%202013-PORT_ 0.pdf

15. Hirshkowitz M, Whiton K, Albert SM, Alessi C, Bruni O, DonCarlos L, et al. National Sleep Foundation's updated sleep duration recommendations: final report. Sleep Health. 2015;1(4):233-243.

16. Felden EPG, Filipin D, Barbosa DG, Andrada RD, Meyer C, Beltrame TS, et al. Adolescentes com sonolência diurna excessiva passam mais tempo em comportamento sedentário. Rev Bras Med Esporte. 2016;22(3):186-190.

17. Sedrez JA, Rosa MIZ, Noll M, Medeiros FS, Candotti CT. Fatores de risco associados a alterações posturais estruturais da coluna vértebra em crianças e adolescentes. Rev Paul Pediatr. 2015;33(1):72-81.

18. Silva FG, Silva CR, Braga LB, Serrão Neto A. Hábitos e problemas do sono dos dois aos dez anos: estudo populacional. Acta Pediatr Port. 2013;44(5):196-202.

19. Santos CIS, Cunha ABN, Braga VP, Saad IAB, Ribeiro MAGO, Conti PBM, et al. Ocorrência de desvios posturais em escolares do ensino público fundamental de Jaguariúna, São Paulo. Rev Paul Pediatr. 2009;27(1):74-80.

20. Preto LSR, Santos ARR, Rodrigues VMCP, Quitério NFN, Pimentel MH, Manrique GA. Análise por Fotogrametria 
da Postura e Fatores de Risco Associados 10 em Crianças e Adolescentes Escolarizados. Paranzini et al Rev Enferm Refer. 2015;4(7):31-40.

21. Detsch C, Luz AMH, Candotti CT, Oliveira DS, Lazaron F, Guimarães LK, et al. Prevalência de alterações posturais em escolares do ensino médio em uma cidade no Sul do Brasil. Rev Panam Salud Publica. 2007;21(4):231-238.

22. Nool M, Candotti CT, Tiggemann CL, Schenell MCW, Vieira A. Prevalência de hábitos posturais inadequados de escolares do ensino fundamental da cidade de Teutônia: um estudo de base populacional. Rev Bras Ciênc Esporte. 2013;35(4):983-1004.

23. Rangel MA, Baptista C, Pitta MJ, Anjo S, Leite AL. Qualidade do sono e prevalência das perturbações do sono em crianças saudáveis em Gaia: um estudo transversal. Rev Port Med Geral Fam. 2015;31(4):256-264.

Recebido: 15/04/2020

Aprovado: 16/06/2021 